\title{
Regulation of Cigarette Smoke (CS)-Induced Autophagy by Nrf2
}

\author{
Lingxiang Zhu ${ }^{1}$, Erika C. Barret ${ }^{1}$, Yuxue $\mathrm{Xu}^{1,2}{ }^{\text {, Zuguo Liu }}{ }^{2}$, Aditya Manoharan ${ }^{1}$, Yin Chen ${ }^{1 *}$ \\ 1 Department of Pharmacology and Toxicology, University of Arizona, Tucson, Arizona, United States of America, 2 School of Medicine, Xiamen University, Xiamen, China
}

\begin{abstract}
Cigarette smoke (CS) has been reported to induce autophagy in airway epithelial cells. The subsequent autophagic cell death has been proposed to play an important pathogenic role in chronic obstructive pulmonary disease (COPD); however, the underlying molecular mechanism is not entirely clear. Using CS extract (CSE) as a surrogate for CS, we found that it markedly increased the expressions of both LC3B-I and LC3B-II as well as autophagosomes in airway epithelial cells. This is in contrast to the common autophagy inducer (i.e., starvation) that increases LC3B-II but reduces LC3B-I. Further studies indicate that CSE regulated LC3B at transcriptional and post-translational levels. In addition, CSE, but not starvation, activated Nrf2-mediated adaptive response. Increase of cellular Nrf2 by either Nrf2 overexpression or the knockdown of Keap1 (an Nrf2 inhibitor) significantly repressed CSE-induced LC3B-I and II as well as autophagosomes. Supplement of NAC (a GSH precursor) or GSH recapitulated the effect of Nrf2, suggesting the increase of cellular GSH level is responsible for Nrf2 effect on LC3B and autophagosome. Interestingly, neither Nrf2 activation nor GSH supplement could restore the repressed activities of mTOR or its downstream effctor-S6K. Thus, the Nrf2-dependent autophagy-suppression was not due to the reactivation of mTOR-the master repressor of autophagy. To search for the downstream effector of Nrf2 on LC3B and autophagosome, we tested Nrf2-dependent genes (i.e., NQO1 and P62) that are also increased by CSE treatment. We found that P62, but not NQO1, could mimic the effect of Nrf2 activation by repressing LC3B expression. Thus, Nrf2->P62 appears to play an important role in the regulation of CSE-induced LC3B and autophagosome.
\end{abstract}

Citation: Zhu L, Barret EC, Xu Y, Liu Z, Manoharan A, et al. (2013) Regulation of Cigarette Smoke (CS)-Induced Autophagy by Nrf2. PLoS ONE 8(4): e55695. doi:10.1371/journal.pone.0055695

Editor: Hong Wei Chu, National Jewish Health, United States of America

Received October 17, 2012; Accepted January 2, 2013; Published April 9, 2013

Copyright: ( 2013 Zhu et al. This is an open-access article distributed under the terms of the Creative Commons Attribution License, which permits unrestricted use, distribution, and reproduction in any medium, provided the original author and source are credited.

Funding: The study was supported by an Arizona Biomedical Research Commission grant, a National Institutes of Health grant RO1Al061695, and a Flight Attendant Medical Institute's (FAMRI) Clinical Innovator Award (CIA). The funders had no role in study design, data collection and analysis, decision to publish, or preparation of the manuscript.

Competing Interests: The authors have declared that no competing interests exist.

*E-mail: ychen@pharmacy.arizona.edu

\section{Introduction}

Autophagy is a regulated catabolic process, by which cellular components are sequestered in the vesicular system and later delivered to lysosomes for degradation and recycling of biogenic components [1]. Autophagy has been demonstrated to play a critical role in maintenance of cellular homeostasis and the adaption to environmental stress such as oxidative stress, starvation, hypoxia and infection [1-4]. The final outcome of autophagy could be either cell death or survival, and its morphological and biomedical features are distinct from other cell death pathway (e.g. apoptosis). A set of autophagy-related genes (ATG) have been identified to be responsible for the regulation of each step of autophagic pathway [5].

Most of the ATG proteins are highly conserved in mammals. Class III phosphoinositide 3-kinase (PI3K) and ATG6 initiate the formation of autophagosomes. Furthermore, two ubiquitinationlike conjugation systems are required for autophagosome formation. One of these systems mediates the conjugation of ATG12 to ATG5 [6], and the other mediates a covalent linkage between LC3B (Atg8) and phosphatidylethanolamine (PE) [7]. An ATG12ATG5 conjugate is present on the outer side of the isolation membrane and is required for elongation of the isolation membrane [8]. A PE-conjugated form of LC3B localizes on the isolation membrane and the autophagosome membrane $[9,10]$.
The unconjugated (designated as LC3B-I) and conjugated forms (designated as LC3B-II) of LC3B can be easily separated by SDSPAGE and detected by antibody staining on the western blot [11]. The intracellular LC3B-II can usually be recognized by its unique "punctate dot" structure [9,11]when the cells are transfected with GFP-LC3B and challenged with autophagy-inducers. Because the level of LC3B-II is generally correlated with the number of autophagosomes [9], the currently established standard [12] uses the amount of LC3B-II (through either in vitro western blot or in vivo punctate formation) as the hallmark and surrogate for autophagic activity.

Despite the overwhelming studies on autophagy in diseases, very few have been carried on non-malignant airway diseases. Recently, several studies have demonstrated that cigarette smoke (CS) or cigarette smoke extract (CSE) induces autophagy in lung cells [13-15], and this autophagic process appears to play a critical role in the pathogenesis of emphysema [13,16]. In these studies, reactive oxygen species (ROS) has been suggested to mediate CSE-induced autophagy, but the detailed mechanism is not entirely clear. Interestingly, it is well established that anti-oxidant systems such as Nrf2 protect the animal from CS-induced lung injury and airway inflammation [17], and the deregulation of Nrf2 contributes to the pathogenesis of emphysema [18-20]. Thus, we speculate that $\mathrm{Nrf2}$ may also have the ability to regulate autophagy in CS/CSE exposure model. 
A)
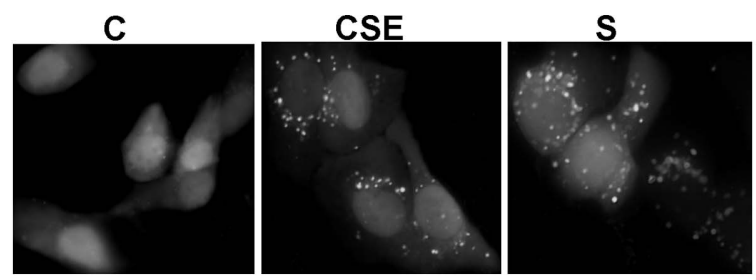

B)

$\frac{2 h}{C \quad S \quad S+3-M A C} \frac{6 h}{S \quad S+3-M A}$

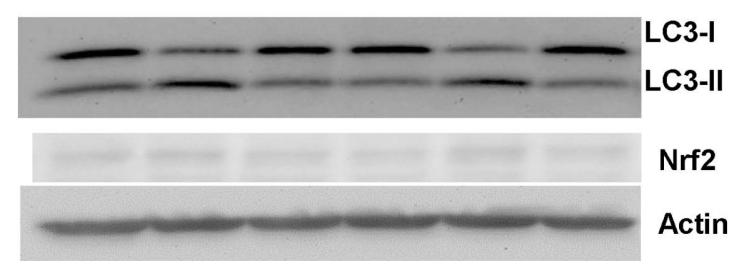

D)

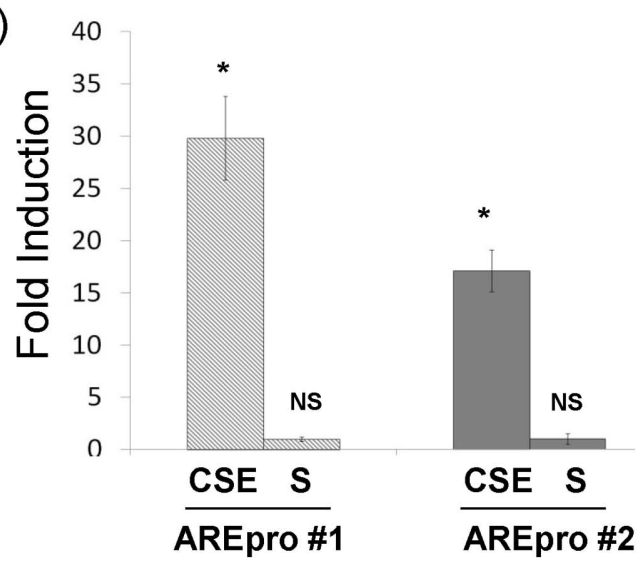

Figure 1. CSE-enhanced autophagy is different from the starvation-induced autophagy in airway epithelial cells. All western blot images were the representative image from at least three independent analyses, and Actin was use as a loading control. A) Increase of cellular autophagosome by live imaging. Cells stably transfected with GFP-LC3B were treated either with $10 \%$ CSE for 24 hrs or were immersed in HBSS buffer (starvation or S) for 6 hrs. Control (C) was sham (medium)-treated sample. Live images were recorded. "punctate dots" of GFP-LC3B were the indication of autophagosomes. B) Starvation induced autophagy but not Nrf2. Cells were immersed in HBSS (S) without or with 3-Methyladenine (3MA). Total protein was collected at the indicated time point and subject to western blot analysis. C) CSE induced both autophagy and Nrf2. Cells were treated with different dose (as indicated in the figure) for either $6 \mathrm{hrs}$ or $24 \mathrm{hrs}$. Total protein was collected and subject to western analysis. D) CSE induced ARE-reporter activation. Two different ARE-reporters were transfected into cells. $24 \mathrm{hrs}$ later, the cells were treated with $10 \%$ CSE for 24 hrs or immersed in HBSS (S) for 6 hrs. Cell lysates were prepared and subject to dual luciferase assay. Relative luciferase unit (RLU) was calculated as described in the Materials and Methods. Fold induction = RLU of treated cells/RLU of control cells. ${ }^{*}: p<0.05, n=5$. NS: not significant.

doi:10.1371/journal.pone.0055695.g001

\section{Materials and Methods}

\section{Chemicals, antibodies, and plasmids}

3-Methyladenine (3-MA), E64D, Pepstatin A were purchased from Sigma-Aldrich (St. Louis, MO). Antibodies against LC3B, p62 and HO-1 were from MBL International (Woburn, MA). Nrf2, Keapl and Actin antibodies were purchased from Santa Cruz Biotechnology (Santa Cruz, CA). Antibodies against pmTOR (Ser 2448) and p-S6K (Thr 389) were purchased from Cell signaling technology (Danvers, MA). The plasmids of Nrf2 and GFP-LC3B were kind gifts from Dr. Jingbo Pi (The Hamner Institutes, RTP, NG) and Dr. Tamotsu Yoshimori (Osaka University, Osaka, Japan), respectively. P62 plasmid and two ARE-reporters (pARE_Gst-luc contains ARE sequence from mouse glutathione S-transferase Ya subunit promoter [21]; pARE_NQO1-luc contains ARE sequence from human NQO1 promoter [22]) were kind gifts from Dr. Donna Zhang (University of Arizona, AZ).

\section{Cell culture}

BEAS-2B was cultivated on regular tissue culture dish in a humidified atmosphere of $5 \% \mathrm{CO}_{2} /$ balanced air at $37^{\circ} \mathrm{C}$ as described before [23]. Before experiments, the cells were cultivated in growth factor free medium for $16 \mathrm{hrs}$.

\section{CSE preparation and treatment}

CSE preparation was based on the protocol described previously with some modifications [15]. Briefly, Kentucky 1R3F research-reference filtered cigarettes (The Tobacco Research Institute, University of Kentucky, Lexington, Kentucky) were smoked using a peristaltic pump (VWR International). Cigarette smoke from three cigarettes was bubbled through $30 \mathrm{ml}$ of F12 medium. This solution was regarded as $100 \%$ strength CSE. pH was adjusted to 7.4 and used within $20 \mathrm{~min}$ after preparation. For the treatment, CSE was diluted directly into the culture medium for the indicated percentage.

\section{Transient transfection and dual luciferase reporter assay}

For the dual-luciferase reporter gene assay, BEAS-2B cells were transfected with the pARE_Gst-luc or pARE_NQO1-luc along with the Renilla luciferase expression plasmid pGL4.74 (hRluc/ TK) (Promega). At $24 \mathrm{hrs}$ post-transfection, the transfected cells were treated with CSE for $24 \mathrm{hrs}$ or immersed in the starve media for $6 \mathrm{hrs}$. Then, the cells were lysed with passive lysis buffer (Promega) and both firefly and Renilla luciferase activities were measured with the dual-luciferase reporter assay system purchased from Promega. Firefly luciferase activity was normalized to Renilla 
A)

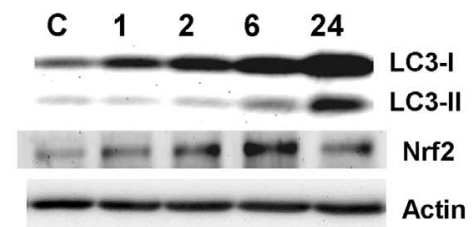

B)

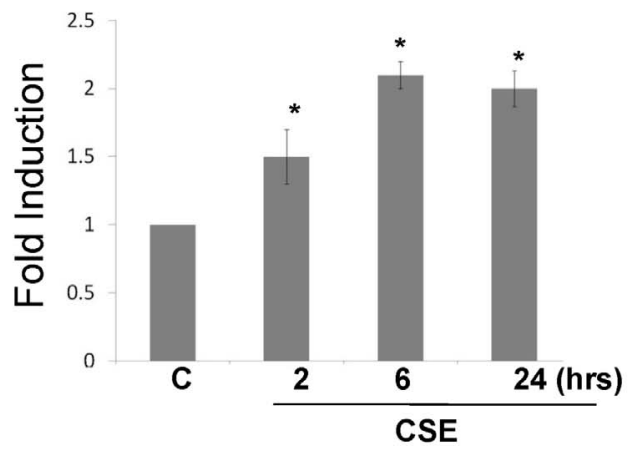

C)

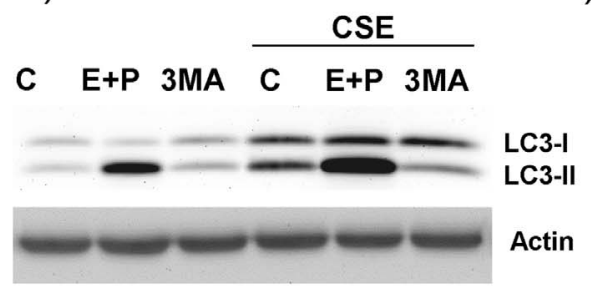

D)

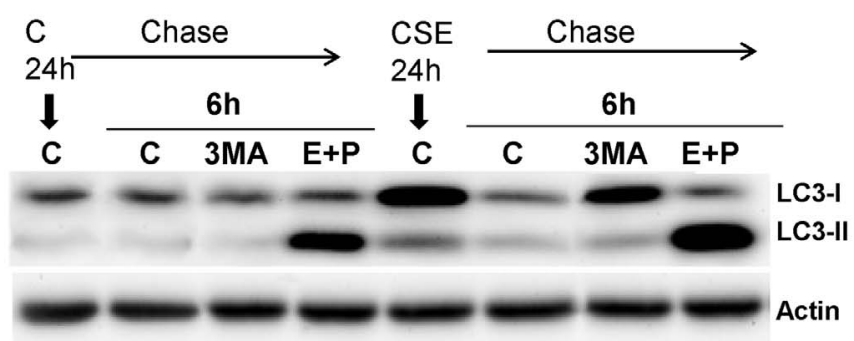

Figure 2. CSE induced LC3B through at multiple levels. All western blot images were the representative image from at least three independent analyses, and Actin was use as a loading control. A) Time course of CSE induced LC3B and Nrf2. Cells were treated with 10\% CSE. At each indicated time point, total protein was collected and subject to western blots analysis. B) CSE increased LC3B steady-state RNA. Cells were treated with $10 \%$ CSE. At each indicated time point, total RNA was collected and subject to Real-time PCR analysis. *: $p<0.05, n=5$. C) CSE regulates LC3B-I and II at posttranslational levels. Cells were treated with 10\% CSE for 24 hrs with either 3-MA (3MA) or lysozymal protease inhibitors-E64D and Pepstatin A (E+P). D) Pulse-chase study. Cells were treated with or without $10 \%$ CSE for 24 hrs. Then, CSE was removed and thoroughly washed. The cells were left alone $(C)$ or treated with various inhibitors: cycloheximide (Cy), 3-MA (3MA) or E64D + Pepstatin A (E+P) for additional 6 hrs. Total protein was collected and subject to western blots analysis. doi:10.1371/journal.pone.0055695.g002

luciferase activity. The experiment was carried out in triplicate and expressed as the mean \pm the standard deviation $(\mathrm{SD})$.

\section{Live imaging of autophagosome}

BEAS-2B cells were transfected with GFP-LC3B. At $24 \mathrm{hrs}$ post-transfection, the cells were treated with or without CSE for $24 \mathrm{hrs}$. Fluorescence Images were acquired by using of a confocal microscope (LSM 510 meta, Carl Zeiss, Thornwood, NY). Six random areas $(20 \mathrm{X})$ were selected for the quantification on each slide, and triplicate samples were included for each experimental group. Cells containing "punctate dot" were counted and expressed as percentage of the total cells.

\section{Western blot}

Total cellular proteins were collected and western blot analysis were performed based on the methods described previously [24]. The sources of antibodies have been described in the "Chemicals, antibodies, and plasmids". Equal protein load was confirmed using the staining of anti - actin antibody.

\section{Real-time $P C R$}

Real-time PCR was performed as described previously [25]. cDNA was prepared from $3 \mu \mathrm{g}$ of total RNA with Moloney murine leukemia virus (MoMLV)-reverse transcriptase (Promega, Inc.) by oligo-d T primers for $90 \mathrm{~min}$ at $42^{\circ} \mathrm{C}$ in a $20-\mu \mathrm{l}$ reaction solution, and was then further diluted to $100 \mu \mathrm{l}$ with water for the following procedures. Two microliters of diluted cDNA were analyzed using 2x SYBR Green PCR Master Mix by an ABI 5700 or ABI Prism 7900HT Sequence Detection System (Applied
Biosystems Inc., Foster City, CA), following the manufacturer's protocol. Primers (Table 1) were used at $0.2 \mu \mathrm{M}$. The PCR reaction was performed in 96-well optical reaction plates, and each well contained a $50-\mu \mathrm{l}$ reaction mixture. The SYBR green dye was measured at $530 \mathrm{~nm}$ during the extension phase. The relative mRNA amount in each sample was calculated based on the $\Delta \Delta \mathrm{C}_{\mathrm{t}}$ method using housekeeping gene $G A P D H$. The purity of amplified product was determined from a single peak of a dissociation curve. Efficiency curves were performed for each gene of interest relative to the housekeeping gene, based on the manufacturer's instructions. Results were calculated as fold induction over control, as described previously [25].

\section{Small interference RNA (siRNA) and transient transfection}

Two different siRNAs were used for the knockdown of each gene. siRNA targeting Nrf2 (\#1: GTAAGAAGCGAGATGTTAA) [26], \#2: AAGAGTATGAGGTGGAAAAAG [27]), Keapl (\#1: GGGCGTGGCTGTCCTCAAT) [26], \#2: TGAAGGGTGCTGTCATGTA [28]) NQO1 (\#1: GGACATCACAGGTAAACTG, \#2: GAACGTCAACTGACATATA [29]) and P62 (\#1: GCATTGAAGTTGATATCGA) [30]) were synthesized by Ambion (Austin, TX). The second set of P62 was the pooled siRNA purchased from Santa Cruz Biotechnology (Santa Cruz, CA). Since the results were identical, only the data from \#1 were presented. In addition, non-targeted control siRNA (siRNA \#1 from Ambion) and individual randomized sequence for Nrf2, Keap1, NQO1 and P62 were also tested as described in the previous publication [31]. Because of identical results, only 
A)

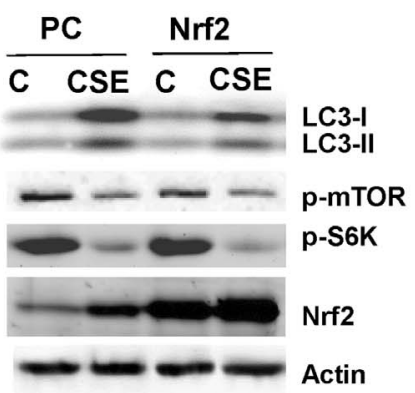

B)

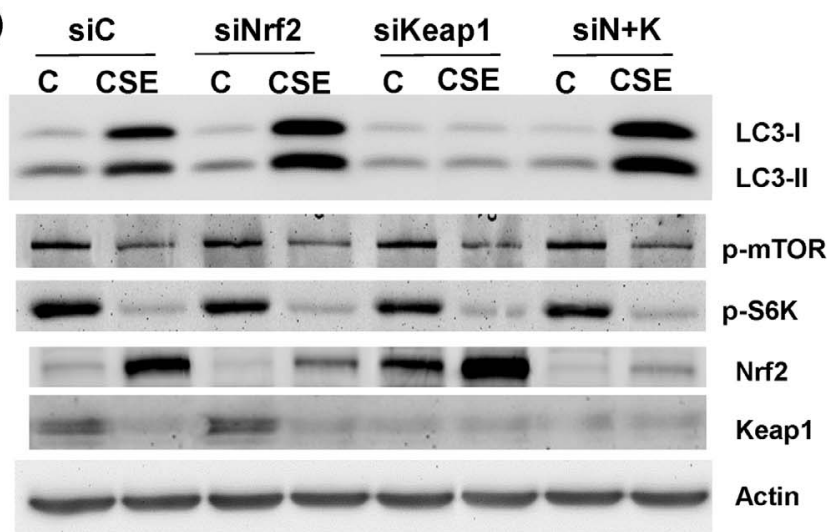

C)

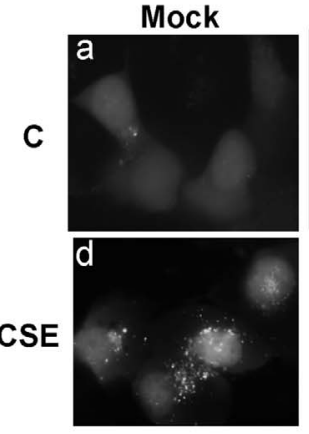

Nrf2
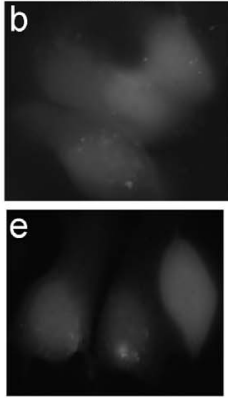

siKeap1

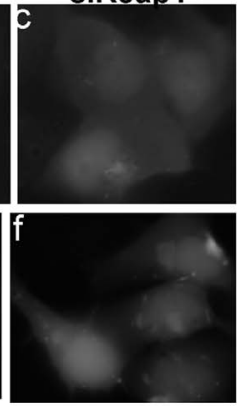

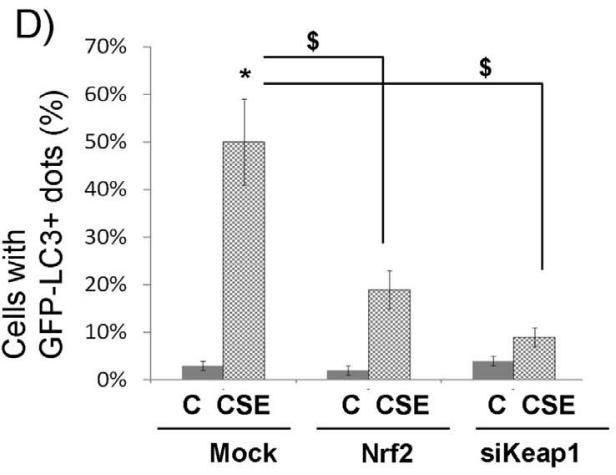

Figure 3. Nrf2 negatively regulate LC3B I and II. All western blot images were the representative image from at least three independent analyses, and Actin was use as a loading control. A) Effect of Nrf2 overexpression. Cells were transfected with Nrf2 plasmid (Nrf2) or empty vector (PCDNA3, or PC). 24 hrs later, cells were treated without (C) or with (CSE) $10 \%$ CSE for 24 hrs. B) Effect of siRNA knockdown of siNrf2 and/or Keap1. Cells were transfected with siRNA control (siC), siRNA against Nrf2 (siNrf2), siRNA against Keap1 (siKeap1), and siNrf2+siKeap1 (siN+K). 24 hrs later, cells were treated without (C) or with (CSE) 10\% CSE for 24 hrs. C) Negative regulation of autophagosome by Nrf2. Cells stably transfected with GFPLC3B were transfected with Nrf2 plasmid (Nrf2) or siRNA against Keap1 (siKeap1). Control was the mock-transfected cells. These cells were treated without (C) or with (CSE) 10\% CSE for 24 hrs. Live images were recorded. "punctate dots" of GFP-LC3B were the indication of autophagosomes. D) Quantification of autophagosome (GFP-LC3B+ dots) was described in Materials and Methods. ${ }^{*}, \$: p<0.05, n=3$. doi:10.1371/journal.pone.0055695.g003

siRNA \#1 was used as control for all the siRNA knockdown studies. siRNA was transfected into cells using lipofectamine ${ }^{\mathrm{TM}}$ 2000 (Invitrogen, Carlsbad, CA) based on manufacturer's instruction. Successful knockdown of the target was confirmed by realtime RT-PCR and western blot.

\section{Measurement of Intracellular Reactive Oxygen Species by Live Cell Imaging}

Reactive oxygen species (ROS) production was determined using chloromethyl-2', $7^{\prime}$-dichlorodihydrofluorescein diacetate (CM-H ${ }_{2}$ DCFDA; Molecular Probes, Eugene, OR) according to the manufacturer's instruction. Briefly, cells were rinsed twice with warm phenol red-free medium and then loaded for $30 \mathrm{~min}$ with CM- $\mathrm{H}_{2}$ DCFDA $(3 \mu \mathrm{M})$ in medium without phenol red. The cells were rinsed three times for removal of the extracellular dye, and the medium was replaced with phenol red-free medium. Then the cells were loaded into a live cell imaging incubator (Carl Zeiss, Thornwood, $\mathrm{NY}$ ) at $37^{\circ} \mathrm{C}$ in a $5 \% \mathrm{CO}_{2}$ atmosphere. Live cell images were continuously recorded before and after CSE treatment by confocal microscopy (LSM 510 meta, Carl Zeiss).

\section{Statistical analysis}

Experimental groups were compared using a two-sided Student's $t$ test, with significance level set as $P<0.05$. When data were not distributed normally, significance was assessed with the Wilcoxon matched-pairs signed-ranks test, and $P<0.05$ was considered to be significant. Matlab 6.0 with statistics toolbox (MathWorks, Inc., Natick, MA) was used for analyses of the data.

\section{Results}

1. CSE induced LC3B-I and II as well as autophagosomes, but CSE-induced autophagy was different from that induced by starvation

Starvation-induced autophagy has been extensively studied. Recently, CSE has also been shown to induce autophagy in airway cells [13-15]. The hallmark of autophagy is the elevation of LC3BII [12]. We compared these two stimuli in the induction of autophagy in airway epithelial cells. Indeed, both starvation and CSE dramatically increased cellular autophagosomes as compared with non-treated control (Fig. 1A). Significant increase of LC3B-II and decrease of LC3B-I, thus the increase of LC3B-II: LC3B-I ratio, was observed in the starved cells at both $2 \mathrm{hrs}$ and $6 \mathrm{hrs}$ (Fig. 1B). This conversion (from LC3B-I to LC3B-II) was significantly blocked by 3-MA (type III PI3K kinase inhibitor) treatment, suggesting that a classical autophagy dependent mechanism is responsible for this process. Starvation for $24 \mathrm{hrs}$ caused significant cell death and the reduction of overall LC3B in these cells (data not shown). Thus, we did not include this time 


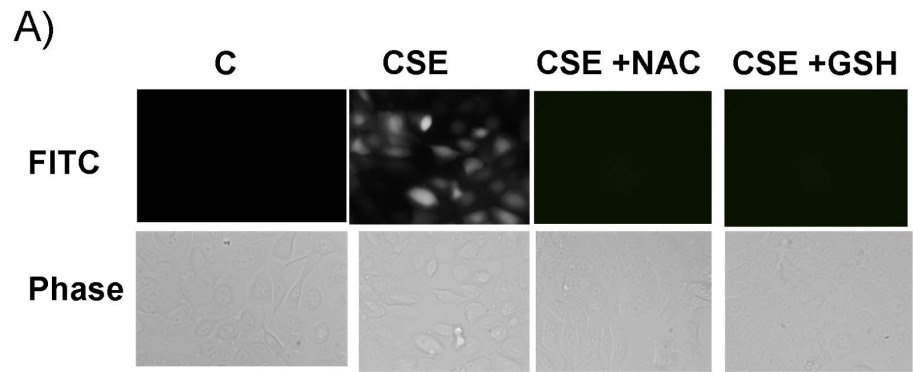

B)

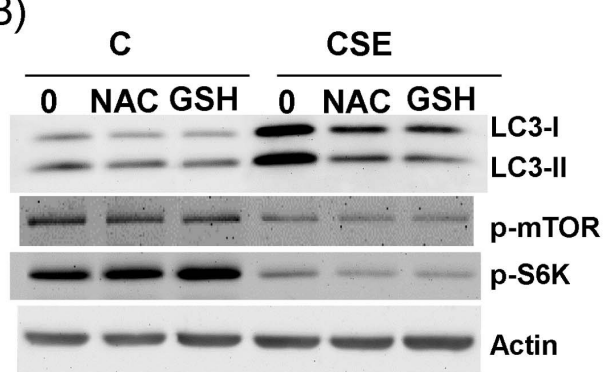

C)

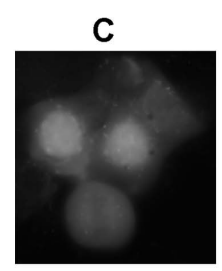

CSE

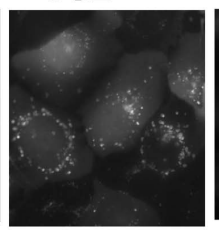

CSE +NAC

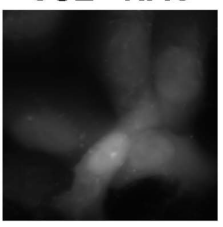

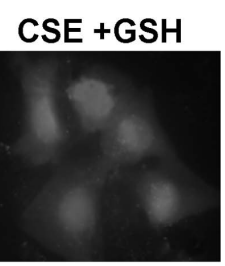

D)

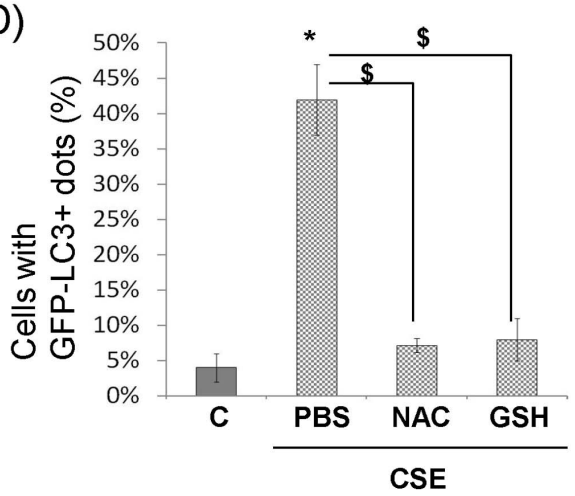

Figure 4. Antioxidant supplement repressed CSE-induced LC3B and autophagosome. A) Antioxidants neutralized CSE-induced cellular ROS. Cells were treated with $10 \%$ CSE or plus NAC (CSE+NAC) or GSH (CSE+GSH). Images were recorded 30 min after dye loading. Green fluorescence indicates generation of reactive oxygen species that oxidized the dye to emit fluorescence. B) Antioxidants downregulated CSE-induced LC3B. C) Antioxidants downregulated CSE-induced autophagosome. D) Quantification of autophagosome (GFP-LC3B+ dots) was described in Materials and Methods. ${ }^{*}, \$: p<0.05, n=3$.

doi:10.1371/journal.pone.0055695.g004

point. Different from starvation, CSE increased both LC3B-I and LC3B-II time- and dose-dependently (Fig. 1C). At lower dose (4\%), CSE treatment slightly induced both LCB-I and II level; but at the higher dose (10\%), LCB-I and II were elevated at $6 \mathrm{hrs}$ and markedly increased at $24 \mathrm{hrs}$. CSE also increased the level of Nrf2 (Fig. 1C), a master regulator of cellular antioxidant response. Nrf2 appeared to be elevated early (6 hrs) and later slightly subsided (24 hrs). Consistent with Nrf2 increase, the activities of two different ARE reporters (pARE_Gst-luc or pARE_NQO1-luc) were significantly increased in CSE treated as compared with nontreated controls (Fig. 1D). The highest dose (i.e. 20\%) appeared to cause outright toxic effects that shut down all the processes. Thus, we used $10 \%$ CSE for the following studies. In contrast, starvation had no effect on Nrf2 (Fig. 1B) or ARE promoter activities (Fig. 1D).

\section{CSE regulated LC3B-I and II at transcriptional and post- translational levels}

Since LC3B is the critical player of autophagy and its dynamics in CSE treated cells were clearly different from the classical autophagy inducer-starvation, we examined the potential molecular mechanism underlying CSE-induced LC3B-I and II. As shown in Fig. 2A, CSE increased LC3B-I as early as 1 hrs; but only starting at $6 \mathrm{hrs}$, LC3B-II was elevated. As comparison, Nrf2 induction by CSE peaked at $6 \mathrm{hrs}$ and decreased at $24 \mathrm{hrs}$. By realtime PCR, we found that LC3B mRNA was moderately (but significantly) increased (Fig. 2B). Since LC3B is known to be degraded in the lysosomal compartment after the fusion between autophagosome and lysosome, we asked the question whether or not the elevation of LC3B protein by CSE was perhaps caused by the blockade of this process. LC3B-I was not changed (or slightly increased) either by the blockade of lysosomal protease activity via the treatment of E64D + pepstatin A (two common lysosomal inhibitors), or by the inhibition of autophagosome formation using 3-MA (Fig. 2C). But, LC3B-II level was significantly increased by $\mathrm{E} 64 \mathrm{D}+$ pepstatin $\mathrm{A}$ and was significantly reduced by 3-MA (Fig. 2C). This observation corroborates the well-established paradigm that LC3B-II level is indeed the surrogate of autophagic activity [12]. In contrast, CSE-induced LC3B-I, which is not a common phenomenon by other autophagy stimuli, was not dependent on autophagy; thus it appears to be a CS-specific phenomenon. We also did a pulse-chase study to further understand the kinetic change of LC3B. The cells were treated with/without CSE for $24 \mathrm{hrs}$, then CSE was removed and the level of LC3B was determined after $6 \mathrm{hrs}$. CSE significantly elevated both LC3B-I and II at $24 \mathrm{hrs}\left(5^{\text {th }}\right.$ lane, Fig. 2D). CSE removal for $6 \mathrm{hrs}$ significantly reduced LC3B-I and II almost to the baseline $\left(6^{\text {th }}\right.$ lane, Fig. 2D), suggesting that this process is reversible. The decrease of LC3B-I (but not LC3B-II) after CSE removal was restored by 3-MA treatment $\left(7^{\text {th }}\right.$ lane, Fig. 2D), suggesting that autophagy was the main mechanism to clear up the increased LC3B protein. Enhanced autophagy flux even after CSE removal was demonstrated by the significantly elevated LC3B-II in $\mathrm{CSE}+\mathrm{E}+\mathrm{P}$ treated cells $\left(8^{\text {th }}\right.$ Lane, Fig. 2D) as compared with E+P treated only cells $\left(4^{\text {th }}\right.$ Lane, Fig. 2D). Therefore, CSE regulates LC3B protein at both transcriptional and post-translational levels. 
A)

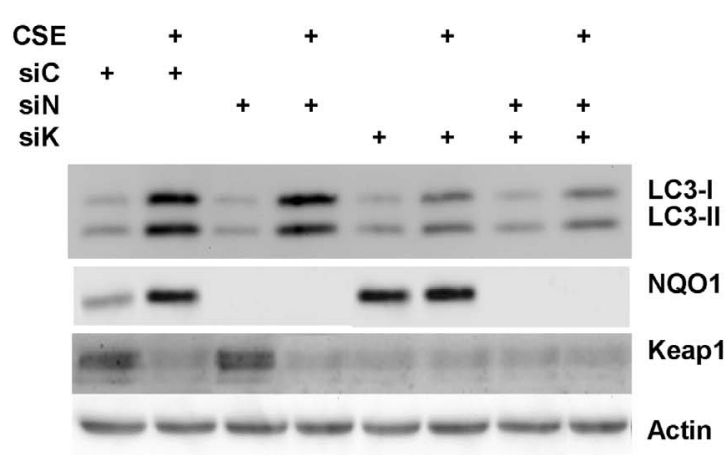

B)

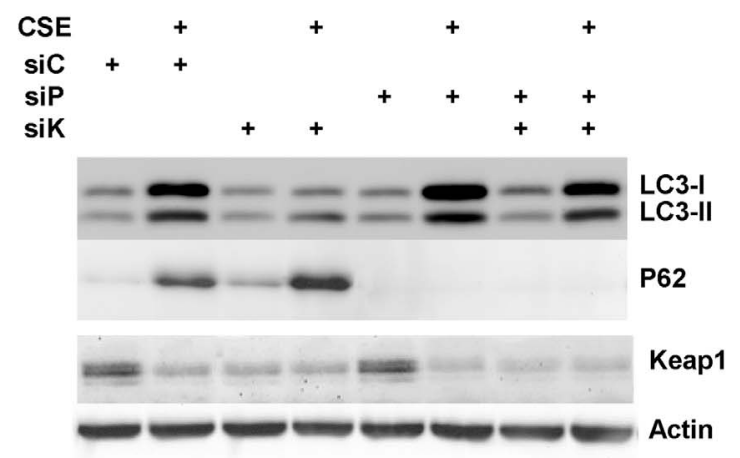

C)

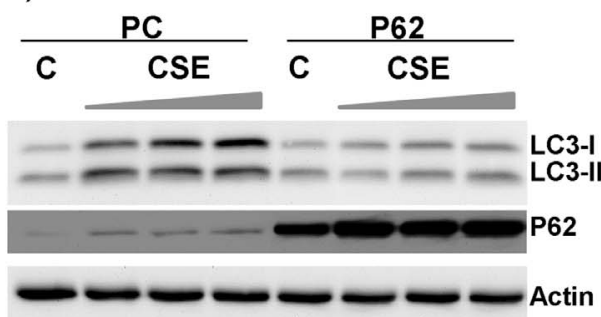

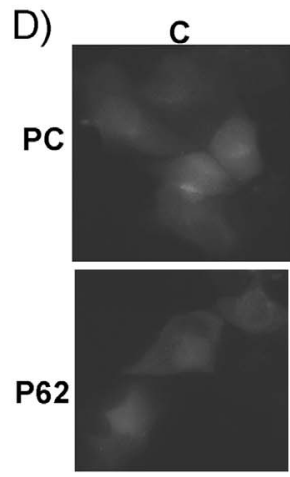

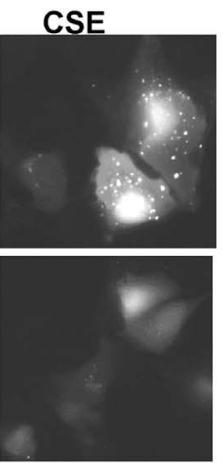

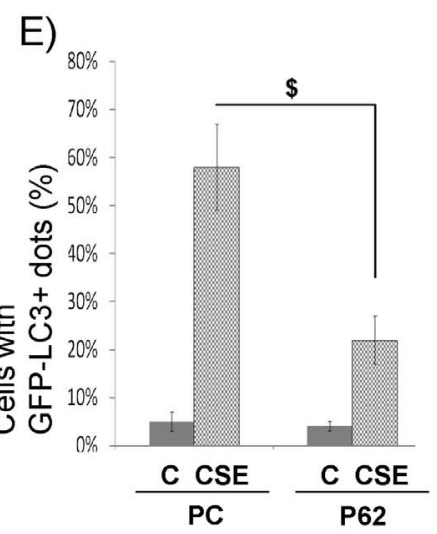

Figure 5. P62, but not NQ01, replicated Nrf2 phenotype. All western blot images were the representative image from at least three independent analyses, and Actin was use as a loading control. A) NQO1 knockdown couldn't rescue Keap1 knockdown. Cells were transfected with siRNA control (siC), siRNA against NQO1 (siN), siRNA against Keap1 (siK), and their combinations as indicated. 24 hrs later, cells were treated without or with $10 \%$ CSE for $24 \mathrm{hrs}$. Total proteins were collected for western blot analysis. B) P62 knockdown couldn't rescue Keap1 knockdown. Cells were transfected with siRNA control (siC), siRNA against P62 (siP), siRNA against Keap1 (siK), and their combinations as indicated. 24 hrs later, cells were treated without or with $10 \%$ CSE for 24 hrs. Total proteins were collected for western blot analysis. C) Effect of P62 overexpression. Cells were transfected with P62 plasmid (P62) or empty vector (PCDNA3, or PC). 24 hrs later, cells were treated without (C) or with (CSE) at increasing doses (8\%, $10 \%, 12 \%)$ for 24 hrs. D) overexpression of P62 represses LC3B. Cells stably transfected with GFP-LC3B were transfected with P62 plasmid (P62) or empty vector (PCDNA3, or PC). These cells were treated without (C) or with (CSE) 10\% CSE for 24 hrs. Live images were recorded. "punctate dots" of GFP-LC3B were the indication of autophagosomes. E) Quantification of autophagosome (GFP-LC3B+ dots) was described in Materials and Methods. *, $\$: p<0.05, n=3$.

doi:10.1371/journal.pone.0055695.g005

\section{Nrf2 negatively regulated LC3B and autophagy, but independent of mTOR}

Since CSE elevated both LC3B and Nrf2, we decided to examine the role of Nrf2 in the regulation of LC3B. We artificially increased Nrf2 level by overexpressing Nrf2. In these cells, LC3B-I and LC3B-II were significantly decreased $\left(4^{\text {th }}\right.$ lane, Fig. 3A) as compared to the mock-transfected cells (2th lane, Fig. 3A). Likewise, increased Nrf2 expression by siRNA knockdown of Keapl (the cellular inhibitor of Nrf2) also repressed both LC3B-I and LC3B-II levels ( $6^{\text {th }}$ lane, Fig. 3B). Note: CSE appeared to significantly repress Keapl expression (Fig. 3B). Conversely, Nrf2 knockdown further increased LC3B-I and II level by CSE $\left(4^{\text {th }}\right.$ lane, Fig. 3B), but not at the non-treatment condition $\left(3^{\text {th }}\right.$ lane, Fig. 3B). In addition, when both $\mathrm{Nrf} 2$ and Keapl were knocked down, the levels of LC3B-I and II were restored to the similar level $\left(8^{\text {th }}\right.$ lane, Fig. 3B) as if Nrf2 was knocked down ( $4^{\text {th }}$ lane, Fig. 3B). Because LC3B-II is the hallmark of autophagy, we examined the impact of Nrf2 on autophagy. As shown in Fig. 3C, overexpression of Nrf2 (3C-e) or siRNA knockdown of Keapl (3C-f) significantly repressed CSE induced autophagosomes. Fig. 3D shows the statistics of Fig. 3C from triplicates. Taken together, Nrf2 activation appears to repress CSE-induced LC3B-I and II as well as autophagosomes.

Since mTOR is the master inhibitor of autophagy, we tested whether or not the repression of CSE-induced LC3B and autophagosome by Nrf2 was due to the activation of mTOR. CSE significantly repressed activated (phosphorylated) mTOR (Fig. 3A-B). The repression of mTOR activity was further corroborated by the decreased phosphorylated S6K (p-S6K) (Fig. 3A-B), a substrate of mTOR. However, neither Nrf2 overexpression nor Keapl knockdown could restore the activations of both mTOR and S6K (Fig. 3A-B). Thus, mTOR is not the target of Nrf2 activation.

\section{Increase of cellular GSH repressed CSE-induced LC3B} and autophagosome but not mTOR

Nrf2 is a master regulator of antioxidant response partly through increasing the cellular reduced glutathione (GSH) level 
via activating several key GSH synthesizing enzymes [17]. Thus, we tested if the enhancement of GSH synthesis could recapitulate the effect of Nrf2 activation. Indeed, when supplementing the cells with $\mathrm{N}$-acetylcysteine (NAC, a GSH precursor) or GSH itself, LC3B I and II were significantly repressed (Fig. 4B). In addition, these supplements repressed cellular ROS generation (Fig. 4A) and CSE-induced autophagosomes (Fig. 4C-D). Similar to Nrf2 activation, NAC or GSH was not able to restore the repressions of mTOR and S6K by CSE (Fig. 4B). Taken together, Nrf2 appears to repress CSE-induced LC3B level and autophagosomes through increasing the cellular antioxidants (e.g. GSH), thereby inhibiting CSE-induced oxidative stress. But, it has no effect on mTOR activity.

\section{P62 is another Nrf2-responsive gene and responsible for repressing CSE-induced LC3B and autophagosomes}

To further understand the molecular mechanism underlying Nrf2-mediated LC3B repression, we searched for downstream effectors of Nrf2. HO-1, an Nrf2 downstream gene, has been shown to modulate CSE-induced LC3B [15]. We extended this finding by examining two additional well-known Nrf2 dependent genes- P62 [32] and NQO1 [33]. They are both elevated by CSE $\left(2^{\text {nd }}\right.$ lane, Fig. $\left.5 \mathrm{~A}-\mathrm{B}\right)$. As shown in the previous study, single knockdown of Keapl repressed LC3B-I and II ( $6^{\text {th }}$ lane, Fig. 3B) while double knockdown of Keapl and Nrf2 could restore CSEinduced LC3B-I and II ( $8^{\text {th }}$ lane, Fig. 3B). Thus, we used the same strategy to determine whether or not either of these two proteins is the downstream effector of Nrf2. As shown in Fig. 5A, double knockdown of Keapl and NQO1 couldn't restore the CSEinduced LC3B-I and II ( $8^{\text {th }}$ lane, Fig. 5A), and single knockdown of NQO1 also had no effect $\left(4^{\text {th }}\right.$ lane, Fig. 5A). In contrast, when both Keapl and P62 were knocked down, CSE-induced LC3B-I and II were restored $\left(8^{\text {th }}\right.$ lane, Fig. 5B). To confirm this observation, we overexpressed $\mathrm{P} 62$ and it indeed repressed the elevations of LC3B-I and II induced by the increasing doses of CSE $\left(6^{\text {th }}-8^{\text {th }}\right.$ lanes, Fig. 5C). Consistently, P62 overexpression also repressed CSE-induced autophagy (Fig. 5D-E). Thus, P62 appears to be the downstream effector of Nrf2 that mediates the repression of CSE-induced LC3B and autophagosomes.

\section{Discussion}

CSE-induced autophagy appears to be different from the autophagy induced by conventional stimuli such as starvation. The conventional stimuli mostly increase the conversion from LC3B-I to LC3B-II, while the overall amount of LC3B appears to be constant. In contrast, CSE significantly increase the total amount of LC3B. The induction of LC3B-I appears to be much earlier than the increase of autophagy (as shown by the increase of LC3BII). However, it is unclear whether CSE accelerates the autophagy biogenesis, which leads to more LC3B-I to LC3B-II conversion; or large amount of LC3B-I induced by CSE drives more LC3B-II

\section{Table 1 Realtime Primers.}

\begin{tabular}{|c|c|}
\hline Gene & Primers \\
\hline \multirow[t]{2}{*}{ LC3B } & forward AGAGCAGCATCCAACCAAAAT \\
\hline & reverse TGAGCTGTAAGCGCCTTCTAA \\
\hline \multirow[t]{2}{*}{ GAPDH } & forward CAATGACCCCTTCATTGACC \\
\hline & reverse GACAAGCTTCCCGTTCTCAG \\
\hline
\end{tabular}

conversion through a concentration-dependent passive mechanism. To investigate these possibilities, we blocked autophagy biogenesis using class III PI3K inhibitor 3-MA. There was no change in LC3B-I and II levels in untreated cells, but 3-MA significantly decreased LC3B-II in CSE-treated cells without affecting LC3B-I. In addition, when lysosomal protease activity was blocked, LC3B-II level was increased. These suggest that CSE did increase the autophagy biogenesis. It is surprising that, even without CSE treatment, LC3B was still recycled through lysozyme but is 3-MA insensitive. This process is perhaps mediated through other non-canonical autophagic pathways. In our pulse/chase study, we further demonstrate that CSE-induced LC3B is reversible. In the CSE-treated cells, autophagosome/lysosomemediated post-translational degradation pathway plays an important role in maintaining LC3B level. The excessive LC3B-I was cleared within $6 \mathrm{hrs}$ by autophagy. The increase of LC3B-I protein (also perhaps mRNA) by CS (or CSE) exposure appears not to be restricted in the lung epithelial cells $[13,15]$. Similar observations have been made in other cell types such as macrophage [14], fibroblast [14], keratinocyte [34], endothelial cells [35], the follicle-associated epithelium and M-cells in peyer patches [36]. Thus, CS (or C.SE) exposure is capable of modulating autophagy in various tissues of the body, and autophagy may play critical role in the pathogenesis of different CS-related illnesses.

In addition to its traditional pro-survival role in the cellular response to stress conditions. excessive autophagy can also lead to cell death (type II) [37]. In the above-mentioned CS (or CSE) induced models, excessive autophagy appears to be largely associated with cellular damage and cell death. Chen $\mathrm{ZH}$ et, al. have elegantly demonstrated that LC3B, through interacting with Fas and Cav, regulates extrinsic apoptosis in CS-induced emphysema model [16]. However, CS (or CSE)-induced autophagy in immune related cells such as macrophage [14] and M cells [36] may have other functions. Indeed, autophagy has been well studied for its broad function in innate and adaptive immunity, including microbicidal activity [38], modulation of pattern recognition receptors [39,40] and antigen presentation [41,42]. Interestingly, microbials can also develop counter measures to hijack autophagic machinery for their own survival. For example, Staphylococcus aureus co-localizes with LC3B and its replication is dependent of autophagy [43]. In addition, Picornavirus (e.g. polio virus and rhinovirus) utilizes the surface of autophagosome as their replicating factory $[44,45]$. Thus, increased autophagosomes by C.S (or CSE) may facilitate the replication and growth of certain viruses or bacteria. To support this speculation, markedly enhanced rhinovirus replication has been demonstrated in the experimental rhinoviral infections on COPD patients [46] or on the epithelial cells derived from the airways of COPD patients [47]. Thus, further studies may be required to completely elucidate the interactions between virus and autophagosome in the context of virus-induced COPD exacerbation [48-50].

Another major distinction between CSE- and starvation-induced autophagy is the involvement of ROS and Nrf2 in the former. Nrf2 has been well established as the master regulator of cellular antioxidant pathway mainly via the activation of a battery of antioxidant gene expression [51]. The importance of Nrf2 in various lung diseases (e.g. acute lung injury, acute respiratory distress syndrome, asthma, COPD, lung cancer, infection etc.) has been extensively reported (reviewed in [51]). Although CSE induces both Nrf2 activation and autophagy, the interaction between these two pathways hasn't been clearly defined. The present study is the first report regarding their interactions in the CS-related models. Previous reports suggest that the lack of 
autophagy causes the accumulation of P62, which leads to persistent activation of Nrf2 via Keapl (the cellular inhibitor of Nrf2) sequestration [22,52]. Thus, Autophagy appears to act upstream to positively regulate Nrf2 activation. In the present study, we have found that the peak Nrf2 activation (6 hrs) occurred early than the peak of autophagy marker-LC3B-II level (24 hrs). The activation of Nrf2 either by overexpression or by Keapl knockdown repressed LC3B-I and II and also decreased autophagosome number. Thus, our study indicates that Nrf2 acts upstream to negatively regulate CS-induced autophagy. Even more intriguingly, we found that $\mathrm{P} 62$ was the downstream effector of Nrf2 that repressed autophagy. Both studies are not entirely conflicting though, because a recent report suggests an Nrf2$>$ p62- $>$ Nrf2 positive loop that regulates Nrf2 mediated antioxidant gene expression [32]. In their model of non-canonical Nrf2 activation, p62 is transcriptionally activated by Nrf2 via ARE site on its promoter; then the accumulated p62 sequesters Keapl and subjects it to autophagic degradation, which leads to persistent Nrf2 activation [32]. However, this model has been established entirely in the non-pulmonary cell types such as HEK293, mouse embryonic fibroblasts, kidney cells and hepatocytes [22,52]. Whether or not it also exists in pulmonary cells require further investigation. Another possibility is that CS-induced autophagy may be different from or be regulated differentially by the canonical autophagy. Previously, Kim HP et al. has demonstrated the protective role of HO-1 in repressing CSE-induced autophagy [15]. This observation supports our finding about the protective role of Nrf2 in CSE-induced autophagy because HO-1 is a classical Nrf2-dependent gene [51]. The present study identifies a second

\section{References}

1. Kundu M, Thompson CB (2008) Autophagy: basic principles and relevance to disease. Annu Rev Pathol 3: 427-455.

2. Kiffin R, Bandyopadhyay U, Cuervo AM (2006) Oxidative stress and autophagy. Antioxid Redox Signal 8: 152-162.

3. Kondo Y, Kanzawa T, Sawaya R, Kondo S (2005) The role of autophagy in cancer development and response to therapy. Nat Rev Cancer 5: 726-734.

4. Mizushima N, Levine B, Cuervo AM, Klionsky DJ (2008) Autophagy fights disease through cellular self-digestion. Nature 451: 1069-1075.

5. Klionsky DJ, Cregg JM, Dunn WA Jr, Emr SD, Sakai Y, et al. (2003) A unified nomenclature for yeast autophagy-related genes. Dev Cell 5: 539-545.

6. Mizushima N, Noda T, Yoshimori T, Tanaka Y, Ishii T, et al. (1998) A protein conjugation system essential for autophagy. Nature 395: 395-398.

7. Ichimura Y, Kirisako T, Takao T, Satomi Y, Shimonishi Y, et al. (2000) A ubiquitin-like system mediates protein lipidation. Nature 408: 488-492.

8. Mizushima N, Yamamoto A, Hatano M, Kobayashi Y, Kabeya Y, et al. (2001) Dissection of autophagosome formation using Apg5-deficient mouse embryonic stem cells. J Cell Biol 152: 657-668.

9. Kabeya Y, Mizushima N, Ueno T, Yamamoto A, Kirisako T, et al. (2000) LC3, a mammalian homologue of yeast Apg8p, is localized in autophagosome membranes after processing. EMBO J 19: 5720-5728.

10. Kabeya Y, Mizushima N, Yamamoto A, Oshitani-Okamoto S, Ohsumi Y, et al. (2004) LC3, GABARAP and GATE16 localize to autophagosomal membrane depending on form-II formation. J Cell Sci 117: 2805-2812.

11. Mizushima N (2004) Methods for monitoring autophagy. Int J Biochem Cell Biol 36: 2491-2502.

12. Klionsky DJ, Abeliovich H, Agostinis P, Agrawal DK, Aliev G, et al. (2008) Guidelines for the use and interpretation of assays for monitoring autophagy in higher eukaryotes. Autophagy 4: 151-175.

13. Chen ZH, Kim HP, Sciurba FC, Lee SJ, Feghali-Bostwick C, et al. (2008) Egr-1 regulates autophagy in cigarette smoke-induced chronic obstructive pulmonary disease. PLoS One 3: e3316.

14. Hwang JW, Chung S, Sundar IK, Yao H, Arunachalam G, et al. (2010) Cigarette smoke-induced autophagy is regulated by SIRT1-PARP-1-dependent mechanism: implication in pathogenesis of COPD. Arch Biochem Biophys 500: 203-209.

15. Kim HP, Wang X, Chen ZH, Lee SJ, Huang MH, et al. (2008) Autophagic proteins regulate cigarette smoke-induced apoptosis: protective role of heme oxygenase-1. Autophagy 4: 887-895.

16. Chen ZH, Lam HC, Jin Y, Kim HP, Cao J, et al. (2010) Autophagy protein microtubule-associated protein 1 light chain-3B (LC3B) activates extrinsic apoptosis during cigarette smoke-induced emphysema. Proc Natl Acad Sci U S A 107: $18880-18885$
Nrf2-dependent gene product-P62 as another autophagy repressor. Thus, Nrf2 system is likely to play an indispensible role in regulating autophagy.

Recently, Fujita, K et, al. indicates that Nrf2-P62 is required for TLR4 mediated aggresome-like induced structure (ALIS) formation [53]. This ALIS has autophagosome-like features such as LC3 positive and being degradable through lysosomal compartment. In their study, Nrf2-P62 appears to be a positive regulator of ALIS formation. However, classical autophagy inhibitors (3-MA and wortmannin) and siRNA knockdown of essential ATG (ATG5, ATG7) failed to prevent this process [53]. Thus, the formation of ALIS is different from the classical autophagy. Nonetheless, this study has reflects the complicated nature of Nrf2-P62 system in controlling a variety of LC3 associated cellular structures that warrant further study.

In summary, we have, for the first time, demonstrated that Nrf2 system negatively regulates CSE-induced LC3B expression and autophagosomes partly through the elevation of P62. The elucidation of this important interaction between Nrf2 systems and autophagy will advance our understanding of the pathogenic effect of CS-associated chronic airway diseases and reveal novel drug targets for the development of effective treatments.

\section{Author Contributions}

Conceived and designed the experiments: YC. Performed the experiments: LZ ECB YX AM. Analyzed the data: YC LZ. Contributed reagents/ materials/analysis tools: ZL. Wrote the paper: YC.

17. Sussan TE, Rangasamy T, Blake DJ, Malhotra D, El-Haddad H, et al. (2009) Targeting Nrf2 with the triterpenoid CDDO-imidazolide attenuates cigarette smoke-induced emphysema and cardiac dysfunction in mice. Proc Natl Acad Sci U S A 106: 250-255.

18. Biswal S, Thimmulappa RK, Harvey CJ (2012) Experimental therapeutics of Nrf2 as a target for prevention of bacterial exacerbations in COPD. Proc Am Thorac Soc 9: 47-51.

19. Sandford AJ, Malhotra D, Boezen HM, Siedlinski M, Postma DS, et al. (2012) NFE2L2 Pathway Polymorphisms and Lung Function Decline in Chronic Obstructive Pulmonary Disease. Physiol Genomics.

20. Suzuki M, Betsuyaku T, Ito Y, Nagai K, Nasuhara Y, et al. (2008) Downregulated NF-E2-related factor 2 in pulmonary macrophages of aged smokers and patients with chronic obstructive pulmonary disease. Am J Respir Cell Mol Biol 39: 673-682.

21. Zhang DD, Hannink M (2003) Distinct cysteine residues in Keapl are required for Keap1-dependent ubiquitination of Nrf2 and for stabilization of Nrf2 by chemopreventive agents and oxidative stress. Mol Cell Biol 23: 8137-8151.

22. Lau A, Wang XJ, Zhao F, Villeneuve NF, Wu T, et al. (2010) A noncanonical mechanism of Nrf2 activation by autophagy deficiency: direct interaction between Keapl and p62. Mol Cell Biol 30: 3275-3285.

23. Chen Y, Zhao YH, Di YP, Wu R (2001) Characterization of human mucin 5B gene expression in airway epithelium and the genomic clone of the aminoterminal and 5'-flanking region. Am J Respir Cell Mol Biol 25: 542-553.

24. Deng J, Chen Y, Wu R (2000) Induction of cell cornification and enhanced squamous-cell marker SPRR1 gene expression by phorbol ester are regulated by different signaling pathways in human conducting airway epithelial cells. Am J Respir Cell Mol Biol 22: 597-603.

25. Kao CY, Chen Y, Thai P, Wachi S, Huang F, et al. (2004) IL-17 markedly upregulates beta-defensin-2 expression in human airway epithelium via JAK and NF-kappaB signaling pathways. J Immunol 173: 3482-3491.

26. Zhu L, Pi J, Wachi S, Andersen ME, Wu R, et al. (2008) Identification of Nrf2dependent airway epithelial adaptive response to proinflammatory oxidanthypochlorous acid challenge by transcription profiling. Am J Physiol Lung Cell Mol Physiol 294: L469-477.

27. Hosoya T, Maruyama A, Kang MI, Kawatani Y, Shibata T, et al. (2005) Differential responses of the Nrf2-Keapl system to laminar and oscillatory shear stresses in endothelial cells. J Biol Chem 280: 27244-27250.

28. Ghosh D, Sachdev S, Hannink M, Roberts RM (2005) Coordinate regulation of basal and cyclic 5'-adenosine monophosphate (cAMP)-activated expression of human chorionic gonadotropin-alpha by Ets-2 and cAMP-responsive element binding protein. Mol Endocrinol 19: 1049-1066. 
29. Alard A, Fabre B, Anesia R, Marboeuf C, Pierre P, et al. (2010) NAD(P)H quinone-oxydoreductase 1 protects eukaryotic translation initiation factor 4GI from degradation by the proteasome. Mol Cell Biol 30: 1097-1105.

30. Pankiv S, Clausen TH, Lamark T, Brech A, Bruun JA, et al. (2007) p62/ SQSTM1 binds directly to Atg8/LC3 to facilitate degradation of ubiquitinated protein aggregates by autophagy. J Biol Chem 282: 24131-24145.

31. Tao S, Zhu L, Lee P, Lee WM, Knox K, et al. (2012) Negative Control of TLR3 Signaling by TICAM1 Down-Regulation. Am J Respir Cell Mol Biol 46: 660667.

32. Jain A, Lamark T, Sjottem E, Larsen KB, Awuh JA, et al. (2010) p62/SQSTM1 is a target gene for transcription factor NRF2 and creates a positive feedback loop by inducing antioxidant response element-driven gene transcription. J Biol Chem 285: 22576-22591.

33. Jaiswal AK (2000) Regulation of genes encoding $\mathrm{NAD}(\mathrm{P}) \mathrm{H}$ : quinone oxidoreductases. Free Radic Biol Med 29: 254-262.

34. Ratovitski EA (2011) DeltaNp63alpha/IRF6 interplay activates NOS2 transcription and induces autophagy upon tobacco exposure. Arch Biochem Biophys 506: $208-215$.

35. Csordas A, Kreutmayer S, Ploner C, Braun PR, Karlas A, et al. (2011) Cigarette smoke extract induces prolonged endoplasmic reticulum stress and autophagic cell death in human umbilical vein endothelial cells. Cardiovasc Res 92: 141148.

36. Verschuere S, Allais L, Bracke KR, Lippens S, De Smet R, et al. (2011) Cigarette smoke and the terminal ileum: increased autophagy in murine follicleassociated epithelium and Peyer's patches. Histochem Cell Biol 137: 293-301.

37. Clarke PG (1990) Developmental cell death: morphological diversity and multiple mechanisms. Anat Embryol (Berl) 181: 195-213.

38. Alonso S, Pethe K, Russell DG, Purdy GE (2007) Lysosomal killing of Mycobacterium mediated by ubiquitin-derived peptides is enhanced by autophagy. Proc Natl Acad Sci U S A 104: 6031-6036.

39. Sanjuan MA, Dillon CP, Tait SW, Moshiach S, Dorsey F, et al. (2007) Toll-like receptor signalling in macrophages links the autophagy pathway to phagocytosis. Nature 450: 1253-1257.

40. Xu Y, Jagannath C, Liu XD, Sharafkhaneh A, Kolodziejska KE, et al. (2007) Toll-like receptor 4 is a sensor for autophagy associated with innate immunity. Immunity 27: 135-144.

41. Schmid D, Pypaert M, Munz C (2007) Antigen-loading compartments for major histocompatibility complex class II molecules continuously receive input from autophagosomes. Immunity 26: 79-92.
42. English L, Chemali M, Duron J, Rondeau C, Laplante A, et al. (2009) Autophagy enhances the presentation of endogenous viral antigens on MHC class I molecules during HSV-1 infection. Nat Immunol 10: 480-487.

43. Schnaith A, Kashkar H, Leggio SA, Addicks K, Kronke M, et al. (2007) Staphylococcus aureus subvert autophagy for induction of caspase-independent host cell death. J Biol Chem 282: 2695-2706.

44. Klein KA, Jackson WT (2011) Human rhinovirus 2 induces the autophagic pathway and replicates more efficiently in autophagic cells. J Virol 85: 96519654.

45. Jackson WT, Giddings TH Jr, Taylor MP, Mulinyawe S, Rabinovitch M, et al. (2005) Subversion of cellular autophagosomal machinery by RNA viruses. PLoS Biol 3: e156.

46. Mallia P, Message SD, Gielen V, Contoli M, Gray K, et al. (2011) Experimental rhinovirus infection as a human model of chronic obstructive pulmonary disease exacerbation. Am J Respir Crit Care Med 183: 734-742.

47. Schneider D, Ganesan S, Comstock AT, Meldrum CA, Mahidhara R, et al. (2010) Increased cytokine response of rhinovirus-infected airway epithelial cells in chronic obstructive pulmonary disease. Am J Respir Crit Care Med 182: 332 340.

48. Tan WC, Xiang X, Qiu D, Ng TP, Lam SF, et al. (2003) Epidemiology of respiratory viruses in patients hospitalized with near-fatal asthma, acute exacerbations of asthma, or chronic obstructive pulmonary disease. Am J Med 115: 272-277.

49. Mallia P, Johnston SL (2006) How viral infections cause exacerbation of airway diseases. Chest 130: 1203-1210.

50. Mallia P, Contoli M, Caramori G, Pandit A, Johnston SL, et al. (2007) Exacerbations of asthma and chronic obstructive pulmonary disease (COPD): focus on virus induced exacerbations. Curr Pharm Des 13: 73-97.

51. Cho HY, Kleeberger SR (2010) Nrf2 protects against airway disorders. Toxicol Appl Pharmacol 244: 43-56.

52. Komatsu M, Kurokawa H, Waguri S, Taguchi K, Kobayashi A, et al. (2010) The selective autophagy substrate p62 activates the stress responsive transcription factor Nrf2 through inactivation of Keap1. Nat Cell Biol 12: 213-223.

53. Fujita K, Maeda D, Xiao O, Srinivasula SM (2011) Nrf2-mediated induction of p62 controls Toll-like receptor-4-driven aggresome-like induced structure formation and autophagic degradation. Proc Natl Acad Sci U S A 108: 1427 1432 